\title{
A new upper bound on the global defensive alliance number in trees*
}

\author{
Xue-gang Chen \\ Department of Mathematics \\ North China Electric Power University \\ Beijing 102206, China \\ Wai Chee Shiu \\ Department of Mathematics \\ Hong Kong Baptist University \\ 224 Waterloo Road, Kowloon Tong, Hong Kong, China
}

Submitted: Oct 17, 2010; Accepted: Oct 3, 2011; Published: Oct 10, 2011

Mathematics Subject Classification: 05C69, 05C35

\begin{abstract}
A global defensive alliance in a graph $G=(V, E)$ is a dominating set $S$ satisfying the condition that for every vertex $v \in S,|N[v] \cap S| \geq|N(v) \cap(V-S)|$. In this note, a new upper bound on the global defensive alliance number of a tree is given in terms of its order and the number of support vertices. Moreover, we characterize trees attaining this upper bound.
\end{abstract}

Keywords: global defensive alliance number, tree, upper bound

\section{Introduction}

Graph theory terminology not presented here can be found in [2]. Let $G=(V, E)$ be a graph with $|V|=n$. The degree, neighborhood and closed neighborhood of a vertex $v$ in the graph $G$ are denoted by $d(v), N(v)$ and $N[v]=N(v) \cup\{v\}$, respectively. The minimum degree and maximum degree of the graph $G$ are denoted by $\delta(G)$ and $\Delta(G)$, respectively. The graph induced by $S \subseteq V$ is denoted by $G[S]$. An endvertex is a vertex which is only adjacent to one vertex. An endvertex in a tree $T$ is also called a leaf, while a support vertex of $T$ is a vertex adjacent to a leaf. Let $L(T)$ denote the set of leaves of $T$. A double star is a tree that contains exactly two vertices that are not endvertices. If

*Corresponding author, email address: wcshiu@hkbu. edu.hk. Research Supported by FRG of HKBU and the Fundamental Research Funds for the Central Universities (10ML39). 
one of these vertices is adjacent to $r$ leaves and the other to $s$ leaves, then we denote this double star by $S(r, s)$.

A set $S$ is called a dominating set if every vertex in $V \backslash S$ has a neighbor in $S$. The domination number of $G$, denoted by $\gamma(G)$, is the minimum cardinality of a dominating set of $G$. A minimum dominating set of a graph $G$ is called a $\gamma(G)$-set.

A set $S$ is called a total dominating set if every vertex in $V$ has a neighbor in $S$. The total domination number of $G$, denoted by $\gamma_{t}(G)$, is the minimum cardinality of a total dominating set of $G$.

In [1] Hedetniemi, Hedetniemi and Kristiansen introduced several types of alliances, including defensive alliance. A non-empty set of vertices $S \subseteq V$ is called a defensive alliance if for every $v \in S,|N[v] \cap S| \geq|N(v) \cap(V \backslash S)|$.

A defensive alliance $S$ is called global if it effects every vertex in $V \backslash S$, that is, every vertex in $V \backslash S$ is adjacent to at least one member of the defensive alliance $S$. In this case, $S$ is a dominating set. The global defensive alliance number $\gamma_{a}(G)$ is the minimum cardinality of a defensive alliance of $G$ that is also a dominating set of $G$. A minimum global defensive alliance of a graph $G$ is called a $\gamma_{a}(G)$-set.

Haynes, Hedetniemi and Henning [2] studied global defensive alliance in graphs. They gave the following results.

Lemma 1.1 (Haynes et. al [2]). For the star $K_{1, r}, \gamma_{a}\left(K_{1, r}\right)=1+\left\lfloor\frac{r}{2}\right\rfloor$.

Lemma 1.2 (Haynes et. al [2]). For the double star $S(r, s), \gamma_{a}(S(r, s))=2+\left\lfloor\frac{r-1}{2}\right\rfloor+\left\lfloor\frac{s-1}{2}\right\rfloor$.

Let $\tau$ be the family of trees $T$, where $T=P_{5}$ or $T=K_{1,4}$ or $T$ is the tree obtained from $t K_{1,4}$ (the disjoint union of $t$ copies of $K_{1,4}$ ) by adding $t-1$ edges between leaves of these copies of $K_{1,4}$ in such a way that the center of each $K_{1,4}$ is adjacent to exactly three leaves in $T$. Haynes et. al established a sharp upper bound on the global defensive alliance number for trees of order greater than 3 .

Lemma 1.3 (Haynes et. al [2]). If $T$ is a tree of order $n \geq 4$, then $\gamma_{a}(T) \leq \frac{3 n}{5}$, with equality if and only if $T \in \tau$.

Chellai and Haynes [3] gave an upper bound on total domination number of a tree in terms of its order and the number of support vertices.

Lemma 1.4 (Chellai and Haynes [3]). If $T$ is a tree of order $n \geq 3$ and with s support vertices, then $\gamma_{t}(T) \leq \frac{n+s}{2}$.

Haynes, Hedetniemi and Henning [2] showed that the global defensive alliance and total domination numbers are the same for graphs with minimum degree at least two and maximum degree at most three.

Lemma 1.5 (Haynes et. al [2]). For any graph $G$ with $\delta(G) \geq 2, \gamma_{t}(G) \leq \gamma_{a}(G)$. Furthermore, if $\Delta(G) \leq 3$, then $\gamma_{t}(G)=\gamma_{a}(G)$.

In this note, a new upper bound on the global defensive alliance number of a tree is given. We show that for a tree of order $n$ and with $s$ support vertices, $\gamma_{a}(G) \leq \frac{n+s}{2}$, and we characterize trees attaining this upper bound. 


\section{Main results}

In order to establish a sharp upper bound on the global defensive alliance number of a tree and to characterize trees achieving this bound, we introduce more notation. For a vertex $v$ in a rooted tree $T$, let $C(v)$ and $D(v)$ denote the sets of children and descendants, respectively, of $v$, and let $D[v]=D(v) \cup\{v\}$.

We introduce a family $\xi$ of trees $T$, where $T$ is a star of odd order or $T$ is the tree obtained from $K_{1,2 t_{1}}, K_{1,2 t_{2}}, \ldots, K_{1,2 t_{s}}$ and $t P_{4}$ (the disjoint union of $t$ copies of $P_{4}$ ) by adding $s+t-1$ edges between leaves of these stars and paths in such a way that the center of each star $K_{1,2 t_{i}}$ is adjacent to at least $1+t_{i}$ leaves in $T$ and each leaf of every copy of $P_{4}$ is incident to at least one new edge, where $t \geq 0, s \geq 2$ and $t_{i} \geq 2$ for $i=1,2, \ldots, s$. Note that each support vertex of each tree in $\xi$ must be adjacent with at least 3 leaves.

Lemma 2.1. Let $T$ be a tree of order $n$ and with $s$ support vertices. If $T \in \xi$, then $\gamma_{a}(T)=\frac{n+s}{2}$.

Proof: Suppose $T$ is a star of odd order. In this case $s=1$. So by Lemma 1.1, we have $\gamma_{a}(T)=\frac{n+s}{2}$. Hence we assume that $T$ is not a star. Let $P_{4}^{1}, P_{4}^{2}, \ldots, P_{4}^{t}$ denote the $t$ disjoint copies of $P_{4}$ when constructing $T$. Let $u_{i}, w_{i}$ be the two support vertices of the path $P_{4}^{i}$. It is obvious that $n=s+2 \sum_{1 \leq i \leq s} t_{i}+4 t$. Let $v_{i}$ be the center of the star $K_{1,2 t_{i}}$ for $i=1,2, \ldots, s$. Let $S$ be a $\gamma_{a}(T)$-set. Since $v_{i}$ is adjacent to at least $1+t_{i}$ leaves in $T$, it follows that $\left|S \cap V\left(K_{1,2 t_{i}}\right)\right| \geq 1+t_{i}$ for $i=1,2, \ldots, s$. In order to dominate $u_{j}, w_{j},\left|S \cap V\left(P_{4}^{j}\right)\right| \geq 2$. Hence, $\gamma_{a}(T)=|S|=\sum_{1 \leq i \leq s}\left|S \cap V\left(K_{1,2 t_{i}}\right)\right|+\sum_{1 \leq j \leq t}\left|S \cap V\left(P_{4}^{j}\right)\right| \geq$ $s+\sum_{1 \leq i \leq s} t_{i}+2 t=\frac{n+s}{2}$. Let $L_{i}$ denote a set of $t_{i}$ leaves of $K_{1,2 t_{i}}$ in $T$ for $i=1,2, \ldots, s$. Let $S^{\prime}=\left\{v_{i} \mid 1 \leq i \leq s\right\} \cup\left\{u_{j}, w_{j} \mid 1 \leq j \leq t\right\} \underset{1 \leq j \leq s}{\bigcup} L_{j}$. Then $S^{\prime}$ is a global defensive alliance of $T$. So, $\gamma_{a}(T) \leq\left|S^{\prime}\right| \leq \frac{n+s}{2}$. Hence, $\gamma_{a}(T)=\frac{n+s}{2}$.

For each tree $T \in \xi$, by its construction, we have the following two simple lemmas.

Lemma 2.2. Let $T \in \xi$. For any $v \in V(T) \backslash L(T)$, there exists a $\gamma_{a}(T)$-set $S$ of $T$ such that $v \in S$ and $|N[v] \cap S|>|N(v) \cap(V(T) \backslash S)|$.

Lemma 2.3. Let $T \in \xi$. For any $v \in V(T) \backslash\left\{v_{1}, v_{2}, \ldots, v_{s}\right\}$, there exists a $\gamma_{a}(T)$-set $S$ of $T$ such that $v \notin S$, where $v_{1}, v_{2}, \ldots, v_{s}$ are defined in the proof of Lemma 2.1.

Theorem 2.4. Let $T$ be a tree of order $n \geq 3$ and with $s$ support vertices. Then $\gamma_{a}(T) \leq$ $\frac{n+s}{2}$, with equality if and only if $T \in \xi$.

Proof: We proceed by induction on $n \geq 3$. If $n=3$, then $T=P_{3}$, and so $\gamma_{a}(T)=2=\frac{n+s}{2}$ and $T \in \xi$. Suppose, then, that for all trees $T^{\prime}$ of order $n^{\prime}$ and with $s^{\prime}$ support vertices, where $3 \leq n^{\prime}<n, \gamma_{a}\left(T^{\prime}\right) \leq \frac{n^{\prime}+s^{\prime}}{2}$ and equality holds if and only if $T^{\prime} \in \xi$. Let $T$ be a tree of order $n$. If $T$ is a star, then, by Lemma 1.1, $\gamma_{a}\left(K_{1, n-1}\right)=1+\left\lfloor\frac{n-1}{2}\right\rfloor \leq \frac{n+s}{2}$ with equality if and only if $n$ is odd. Hence $T \in \xi$. If $T$ is a double star, then it follows from Lemma 1.2 that $\gamma_{a}(T)<\frac{n+s}{2}$. Hence we may assume that $\operatorname{diam}(T) \geq 4$. 
Let $S(T)$ be the set of support vertices of $T$. Suppose that there exists $v \in S(T)$ such that $d(v)=2$. Let $N(v) \cap L(T)=\left\{v^{\prime}\right\}$ and $N(v) \backslash\left\{v^{\prime}\right\}=\{u\}$. Choose $T_{0}=T-\left\{v, v^{\prime}\right\}$. Let $T_{0}$ have order $n_{0}$ and $s_{0}$ support vertices. Then $n=n_{0}+2$. Since $\operatorname{diam}(T) \geq 4$, it follows that $n_{0} \geq 3$. Applying the induction hypothesis to $T_{0}, \gamma_{a}\left(T_{0}\right) \leq \frac{n_{0}+s_{0}}{2}$. Let $S_{0}$ be a $\gamma_{a}\left(T_{0}\right)$-set. If $u \notin S_{0}$, let $S_{0}^{\prime}=S_{0} \cup\left\{v^{\prime}\right\}$, while if $u \in S_{0}$, let $S_{0}^{\prime}=S_{0} \cup\{v\}$. Then, $S_{0}^{\prime}$ is a global defensive alliance of $T$. So $\gamma_{a}(T) \leq\left|S_{0}\right|+1 \leq \frac{n_{0}+s_{0}}{2}+1=\frac{n+s_{0}}{2}$. Since $s_{0} \leq s$, it follows that $\gamma_{a}(T) \leq \frac{n+s}{2}$. Furthermore, suppose $\gamma_{a}(T)=\frac{n+s}{2}$. Then $s_{0}=s$ and $\gamma_{a}\left(T_{0}\right)=\frac{n_{0}+s_{0}}{2}$. By the induction hypothesis, $T_{0} \in \xi$. Since $s_{0}=s$, it follows that $d_{T}(u)=2$ and $x \notin S(T)$, where $x \in N(u) \backslash\{v\}$. Hence, $x$ is a support of $T_{0}$ and is adjacent to only one leaf in $T_{0}$, which is a contradiction. So, $\gamma_{a}(T)<\frac{n+s}{2}$. In the following, we may assume that $d_{T}(v) \geq 3$ for any $v \in S(T)$.

Choose $v$ having the smallest degree among all support vertices of $T$ of eccentricity $\operatorname{diam}(T)-1$. Let $r$ be a vertex at distance $\operatorname{diam}(T)-1$ from $v$. View $T$ as the rooted tree at $r$. Let $u$ denote the parent of $v$, and $x$ the parent of $u$. Let $|C(v)|=l$. Then $l \geq 2$.

Case 1: Suppose that $d_{T}(u) \geq 3$. Let $T_{1}=T-D[v]$. Assume that $T_{1}$ is of order $n_{1}$ and has $s_{1}$ support vertices. Then $n=n_{1}+l+1$ and $n_{1} \geq 3$. By the induction hypothesis we have $\gamma_{a}\left(T_{1}\right) \leq \frac{n_{1}+s_{1}}{2}$. Among all $\gamma_{a}\left(T_{1}\right)$-sets, let $S_{1}$ be chosen to contain the vertex $u$, if possible.

If $u \in S_{1}$, then by adding $v$ and $\left\lfloor\frac{l-1}{2}\right\rfloor$ children of $v$ to $S_{1}$ produces a global defensive alliance of $T$. So $\gamma_{a}(T) \leq\left|S_{1}\right|+1+\left\lfloor\frac{l-1}{2}\right\rfloor \leq \frac{n-1-l+s_{1}}{2}+\frac{l+1}{2}=\frac{n+s_{1}}{2}$. Since $s_{1}<s$, it follows that $\gamma_{a}(T)<\frac{n+s}{2}$. Hence we may assume that $u \notin S_{1}$.

If $u$ has a child $v^{\prime}$ different from $v$ that is a support vertex, then $\left|C\left(v^{\prime}\right)\right| \geq 2$. Since $u \notin S_{1}$, either $C\left(v^{\prime}\right) \subset S_{1}$ or $v^{\prime} \in S_{1}$. For both cases, we can choose another global defensive alliance of $T$ containing $u$ and $v^{\prime}$, contrary to our choice of $S_{1}$. Hence we assume that every child of $u$ different from $v$ is a leaf.

If $u$ is adjacent to more than one leaf, then we can choose a $\gamma_{a}(T)$-set containing $u$, contrary to our choice of $S_{1}$. So we assume $d_{T}(u)=3$ and the child $v^{\prime}$ of $u$ different from $v$ is a leaf. Thus, $v^{\prime} \in S_{1}$. Delete $v^{\prime}$ from $S_{1}$, add $u$, $v$ and $\left\lfloor\frac{l-1}{2}\right\rfloor$ children of $v$ to $S_{1}$ to get a global defensive alliance of $T$. So $\gamma_{a}(T) \leq\left|S_{1}\right|+1+\left\lfloor\frac{l-1}{2}\right\rfloor \leq \frac{n-1-l+s_{1}}{2}+\frac{l+1}{2}=\frac{n+s_{1}}{2}$. Since $s_{1}<s$, it follows that $\gamma_{a}(T)<\frac{n+s}{2}$.

Case 2: Suppose that $d_{T}(u)=2$. Let $T_{2}=T-D[u]$. Assume that $T_{2}$ has order $n_{2}$ and $s_{2}$ support vertices. Then $n=n_{2}+l+2$. Since $\operatorname{diam}(T) \geq 4$, it follows from our choice of $v$ that $n_{2} \geq 3$. By the induction hypothesis we have $\gamma_{a}\left(T_{2}\right) \leq \frac{n_{2}+s_{2}}{2}$. Let $S_{2}$ be a $\gamma_{a}\left(T_{2}\right)$-set. Let $w$ be the parent of $x$.

Case 2.1: $\quad$ Suppose that $d_{T}(x) \geq 3$ or $d_{T}(x)=2$ and $w \in S(T)$. Then $s=s_{2}+1$. Add $u, v$ and $\left\lfloor\frac{l-1}{2}\right\rfloor$ children of $v$ to $S_{2}$ to obtain a global defensive alliance of $T$. So $\gamma_{a}(T) \leq\left|S_{2}\right|+2+\left\lfloor\frac{l-1}{2}\right\rfloor \leq \frac{n-2-l+s_{2}}{2}+\frac{l+3}{2}=\frac{n+s_{2}+1}{2}=\frac{n+s}{2}$.

Furthermore, suppose $\gamma_{a}(T)=\frac{n+s}{2}$. Then $l$ is odd and $\gamma_{a}\left(T_{2}\right)=\frac{n_{2}+s_{2}}{2}$. By the induction hypothesis, $T_{2} \in \xi$. Suppose that $T_{2}=K_{1,2 t}$, for some $t \geq 1$. 
Suppose $x$ is the center of $K_{1,2 t}$. The set consisting of $\{u, v, x\}, t-1$ children of $x$ and $\frac{l-1}{2}$ children of $v$ forms a global defensive alliance of $T$. So $\gamma_{a}(T) \leq$ $3+(t-1)+\frac{l-1}{2}=\frac{2 t+l+3}{2}=\frac{n}{2}<\frac{n+s}{2}$, which is a contradiction. Hence, $x$ is a leaf of star $K_{1,2 t}$. Since the degree of any support vertex of $T$ is at least three, it follows that $t \geq 2$. Then $T$ is a tree obtained from $K_{1,2 t}$ and $K_{1, l+1}$ by adding an edge between one leaf of each star. Then $T \in \xi$.

So we may assume that $T_{2}$ is not a star. Choose $S_{2}$ to be a $\gamma_{a}\left(T_{2}\right)$-set that contains all centers of stars, all leaves of stars that are incident to added edges when constructing $T_{2}$ and support vertices of all copies of $P_{4}$. Since the center of each star is adjacent to at least $1+t_{i}$ leaves in $T_{2}, S_{2}$ contains at least one leaf of each star $K_{1,2 t_{i}}$.

Suppose $x$ is the center of some $K_{1,2 t_{i}}$ for some $i$. Let $y \in N(x) \cap S_{2} \cap L\left(T_{2}\right)$. Then add $u, v$ and $\frac{l-1}{2}$ children of $v$ to $S_{2} \backslash\{y\}$ to get a global defensive alliance of $T$. We have $\gamma_{a}(T) \leq\left|S_{2}\right|+1+\frac{l-1}{2}=\frac{n_{2}+s_{2}}{2}+\frac{l+1}{2}<\frac{n+s}{2}$, which is a contradiction.

Suppose that $x$ is a support vertex of $P_{4}^{i}=l_{i} u_{i} w_{i} m_{i}$, say $x=u_{i}$ for some $i$. Choose $S^{*}$ to be a $\gamma_{a}\left(T_{2}\right)$-set that contains all centers of stars and all end vertices of added edges when constructing $T_{2}$.

Let $T^{\prime}$ be the subgraph of $T_{2}$ induced by the vertices of all copies of $P_{4}$ when constructing $T_{2}$. Let $T^{\prime \prime}$ be the component of $T^{\prime}-l_{i} u_{i}$ that contains vertex $l_{i}$. For each subgraph $P_{4}^{k}$ of $T^{\prime \prime}$, label $P_{4}^{k}$ with $l_{k} u_{k} w_{j} m_{k}$ such that $d_{T_{2}}\left(m_{k}, l_{i}\right)<$ $d_{T_{2}}\left(l_{k}, l_{i}\right)$. Let $S^{* *}$ be obtained from $S^{*} \backslash\left\{l_{i}\right\}$ by replacing $l_{k}$ with $w_{k}$ for each subgraph $P_{4}^{k}$ of $T^{\prime \prime}$. Then add $u, v$ and $\frac{l-1}{2}$ children of $v$ to $S^{* *}$ to get a global defensive alliance of $T$. So $\gamma_{a}(T) \leq\left|S^{* *}\right|+2+\frac{l-1}{2}=\left|S^{*}\right|+1+\frac{l-1}{2}=$ $\frac{n_{2}+s_{2}}{2}+\frac{l+1}{2}<\frac{n+s}{2}$, which is a contradiction.

Suppose that $x$ is a leaf of a star when constructing $T_{2}$. Let $v_{i}$ be the center of the star $K_{1,2 t_{i}}$ in $T_{2}$ that contains $x$ for some $i$. Suppose $\left|N_{T}\left(v_{i}\right) \cap L(T)\right|<1+t_{i}$. Let $S^{*}$ be a $\gamma_{a}\left(T_{2}\right)$-set containing $x$, all centers of stars and all end vertices of added edges when constructing $T_{2}$. Let $S$ be obtained from $\left(S^{*} \backslash V\left(K_{1,2 t_{i}}\right)\right) \cup$ $\left(N_{T}\left(v_{i}\right) \cap L(T)\right)$ by adding $u, v$ and $\frac{l-1}{2}$ children of $v$. Then $S$ is a global defensive alliance of $T$ with cardinality less than $\frac{n+s}{2}$. It is a contradiction. So $\left|N_{T}\left(v_{i}\right) \cap L(T)\right| \geq 1+t_{i}$ and hence $T \in \xi$.

Suppose $x$ is a leaf of $P_{4}^{i}$ for some $i$. Then clearly $T \in \xi$.

Case 2.2: $\quad$ Suppose $d_{T}(x)=2$ and $w \notin S(T)$. Let $T_{3}=T-D[x]$. Assume that $T_{3}$ has order $n_{3}$ and $s_{3}$ support vertices. Then $n=n_{3}+l+3$ and $n_{3} \geq 3$. Applying the induction hypothesis to $T_{3}, \gamma_{a}\left(T_{3}\right) \leq \frac{n_{3}+s_{3}}{2}$. Let $S_{3}$ be a $\gamma_{a}\left(T_{3}\right)$ set.

Subcase 1: Suppose that $d_{T}(w) \geq 3$. Then $s_{3}=s-1$.

i) Suppose $w \notin S_{3}$, or $w \in S_{3}$ and $\left|N_{T_{3}}[w] \cap S_{3}\right|>\left|N_{T_{3}}(w) \cap\left(V\left(T_{3}\right)-S_{3}\right)\right|$. By adding $u$ and $v$ and $\left\lfloor\frac{l-1}{2}\right\rfloor$ children of $v$ to $S_{3}$ produces a global defensive 
alliance of $T$. So $\gamma_{a}(T) \leq\left|S_{3}\right|+2+\left\lfloor\frac{l-1}{2}\right\rfloor \leq \frac{n-3-l+s_{3}}{2}+\frac{l+3}{2}=\frac{n+s_{3}}{2}<\frac{n+s}{2}$.

ii) Now we assume that $w \in S_{3}$ and $\left|N_{T_{3}}[w] \cap S_{3}\right|=\left|N_{T_{3}}(w) \cap\left(V\left(T_{3}\right)-S_{3}\right)\right|$ for all $\gamma_{a}\left(T_{3}\right)$-set $S_{3}$. Suppose there exists $v^{\prime} \in S\left(T_{3}\right) \cap D[w]$ such that $d_{T}\left(w, v^{\prime}\right)=3$. Let $w x^{\prime} u^{\prime} v^{\prime}$ denote the $w-v^{\prime}$ path in $T$. By a similar way as above, we may assume that $d_{T}\left(x^{\prime}\right)=d_{T}\left(u^{\prime}\right)=2$. Let $\left|C\left(v^{\prime}\right)\right|=l^{\prime}$. Since $\left|N_{T_{3}}[w] \cap S_{3}\right|=\left|N_{T_{3}}(w) \cap\left(V\left(T_{3}\right)-S_{3}\right)\right|$, it follows that $d_{T}(w) \geq 4$. Let $T_{31}=T-D[x]-D\left[x^{\prime}\right]$. Let $T_{31}$ have order $n_{31}$ and $s_{31}$ support vertices. Then $n=n_{31}+l+l^{\prime}+6$ and $s_{31}=s-2$. Since $n_{31} \geq 3$, by applying the induction hypothesis to $T_{31}, \gamma_{a}\left(T_{31}\right) \leq \frac{n_{31}+s_{31}}{2}$. Let $S_{31}$ be a $\gamma_{a}\left(T_{31}\right)$-set. Then by adding $\left\{u, v, u^{\prime}, v^{\prime}, x\right\},\left\lfloor\frac{l-1}{2}\right\rfloor$ children of $v$ and $\left\lfloor\frac{l^{\prime}-1}{2}\right\rfloor$ children of $v^{\prime}$ to $S_{31}$ produces a global defensive alliance of $T$. So $\gamma_{a}(T) \leq\left|S_{31}\right|+5+\left\lfloor\frac{l-1}{2}\right\rfloor+\left\lfloor\frac{l^{\prime}-1}{2}\right\rfloor \leq \frac{n-6-l-l^{\prime}+s_{31}}{2}+\frac{l+l^{\prime}+8}{2}=\frac{n+s}{2}$. For the sake of contradiction, suppose we have equality throughout this inequality chain. In particular, $\gamma_{a}(T)=\frac{n+s}{2}, l$ and $l^{\prime}$ are odd, and $\gamma_{a}\left(T_{31}\right)=\frac{n_{31}+s_{31}}{2}$. So, $T_{31} \in \xi$. Since $w \notin S(T), w \notin\left\{v_{1}, v_{2}, \ldots, v_{s}\right\}$. By Lemma 2.3, we can choose a $\gamma_{a}\left(T_{31}\right)$-set $S^{*}$ such that $w \notin S^{*}$. By adding $\left\{u, v, u^{\prime}, v^{\prime}\right\}$, $\frac{l-1}{2}$ children of $v$ and $\frac{l^{\prime}-1}{2}$ children of $v^{\prime}$ to $S^{*}$ produces a global defensive alliance of $T$. Hence $\gamma_{a}(T) \leq\left|S^{*}\right|+4+\frac{l-1}{2}+\frac{l^{\prime}-1}{2}<\frac{n+s}{2}$, which is a contradiction. So in this case $\gamma_{a}(T)<\frac{n+s}{2}$.

We may assume that $d_{T}\left(w, v^{\prime}\right) \leq 2$ for any $v^{\prime} \in S\left(T_{3}\right) \cap D[w]$. Suppose $v^{\prime} \notin S_{3}$. Then $N\left(v^{\prime}\right) \cap L\left(T_{3}\right) \subseteq S_{3}$. Choose a vertex $u^{\prime} \in N\left(v^{\prime}\right) \cap L\left(T_{3}\right)$. Then $\left(S_{3} \backslash\left\{u^{\prime}\right\}\right) \cup\left\{v^{\prime}\right\}$ is a global defensive alliance of $T_{3}$. So, we obtain a global defensive alliance of $T_{3}$, still say $S_{3}$, such that $S\left(T_{3}\right) \cap D[w] \subseteq S_{3}$. Let $p$ be the parent of $w$. Suppose there is a vertex $q \in N(w) \backslash\{p\}$ and $q \notin S_{3}$. Then $q \notin S\left(T_{3}\right)$. Let $q^{\prime} \in N(q) \cap S\left(T_{3}\right)$. Then $q^{\prime} \in S_{3}$. Since $q^{\prime}$ is adjacent to at least two leaves, there exists a vertex $k \in S_{3} \cap L\left(T_{3}\right) \cap C\left(q^{\prime}\right)$. Then $\left(S_{3} \backslash\{k\}\right) \cup\left\{q^{\prime}\right\}$ is a global defensive alliance of $T$. So, we can get another global defensive alliance of $T_{3}$, say $S_{3}^{\prime}$, such that $N(w) \backslash\{p\} \subseteq S_{3}^{\prime}$. Then $w \in S_{3}^{\prime}$ and $\left|N_{T_{3}}[w] \cap S_{3}^{\prime}\right|>\left|N_{T_{3}}(w) \cap\left(V\left(T_{3}\right)-S_{3}^{\prime}\right)\right|$, contradicting our choice of $w$.

Subcase 2: Suppose that $d_{T}(w)=2$. Let $T_{4}=T-D[w]$. Let $T_{4}$ of order $n_{4}$ and $s_{4}$ support vertices. Then $n=n_{4}+l+4$. By the choice of $v$ we have $n_{4} \geq 3$. Applying the induction hypothesis to $T_{4}$, we have $\gamma_{a}\left(T_{4}\right) \leq \frac{n_{4}+s_{4}}{2}$. Let $S_{4}$ be a $\gamma_{a}\left(T_{4}\right)$-set. Let $p$ be the parent of $w$. Suppose that $d_{T}(p) \geq 3$. Then $s_{4}=s-1$. If $p \in S_{4}$, then adding $\{w, u, v\}$ and $\left\lfloor\frac{l-1}{2}\right\rfloor$ children of $v$ to $S_{4}$ produces a global defensive alliance of $T$. If $p \notin S_{4}$, then adding $\{x, u, v\}$ and $\left\lfloor\frac{l-1}{2}\right\rfloor$ children of $v$ to $S_{4}$ produces a global defensive alliance of $T$. So $\gamma_{a}(T) \leq$ $\left|S_{4}\right|+3+\left\lfloor\frac{l-1}{2}\right\rfloor \leq \frac{n-4-l+s_{4}}{2}+\frac{l+5}{2}=\frac{n+s}{2}$. Furthermore, suppose $\gamma_{a}(T)=\frac{n+s}{2}$. Then $l$ is odd and $\gamma_{a}\left(T_{4}\right)=\frac{n_{4}+s_{4}}{2}$. So, $T_{4} \in \xi$. By Lemma 2.2, we can choose a $\gamma_{a}\left(T_{4}\right)$-set $S^{*}$ such that $p \in S^{*}$ with $\left|N_{T_{4}}[p] \cap S^{*}\right|>\left|N_{T_{4}}(p) \cap\left(V\left(T_{4}\right)-S^{*}\right)\right|$. Then adding $\{u, v\}$ and $\frac{l-1}{2}$ children of $v$ to $S^{*}$ produces a global defensive alliance of $T$. So $\gamma_{a}(T) \leq\left|S^{*}\right|+2+\frac{l-1}{2} \leq \frac{n+s-2}{2}<\frac{n+s}{2}$, which is impossible. 
Hence, $\gamma_{a}(T)<\frac{n+s}{2}$.

Now we assume that $d_{T}(p)=2$. Let $q$ be the parent of $p$. Let $T_{5}$ be obtained from $T$ by deleting vertices of $\{p, w, x, u\}$ and adding edge $q v$. Let $T_{5}$ have order $n_{5}$ and support vertices number $s_{5}$. It follows that $n_{5} \geq 3$. Applying the inductive hypothesis to $T_{5}, \gamma_{a}\left(T_{5}\right) \leq \frac{n_{5}+s_{5}}{2}$. Let $S_{5}$ be a $\gamma_{a}\left(T_{5}\right)$-set. Then $n=n_{5}+4$ and $s=s_{5}$. Then $S_{5} \cup\{p, u\}, S_{5} \cup\{p, w\}$ or $S_{5} \cup\{w, x\}$ is a global defensive alliance of $T$. Hence, $\gamma_{a}(T) \leq\left|S_{5}\right|+2 \leq \frac{n-4+s_{5}}{2}+2=\frac{n+s}{2}$. Furthermore, suppose $\gamma_{a}(T)=\frac{n+s}{2}$. Then $l$ is odd, $\gamma_{a}\left(T_{5}\right)=\frac{n_{5}+s_{5}}{2}$ and $T_{5} \in \xi$. Hence $T \in \xi$.

\section{References}

[1] S.M. Hedetniemei, S.T. Hedetniemi and P. Kristiansen, Alliances in graphs, J. Combin. Math. Combin. Comput., 48 (2004), 157-177.

[2] T.W. Haynes, S.T. Hedetniemi and M.A. Henning, Global defensive alliances in graphs, Electronic J. Combin., 10 (2003), \#R47.

[3] M. Chellali and T.W. Haynes, Total and paired domination numbers of a tree, AKCE J. Graphs Combin., 1:2 (2004), 69-75. 